This is an author produced version of a paper published in European journal of pain (London, England). This paper has been peer-reviewed but does not include the final publisher proof-corrections or journal pagination.

Citation for the published paper:

Jakobsson, Ulf and Horstmann, Vibeke

"Psychometric evaluation of multidimensional pain inventory

(Swedish version) in a sample of elderly people"

Eur J Pain. 2005 Nov 18; [Epub ahead of print]

http://dx.doi.org/10.1016/j.ejpain.2005.10.004

Access to the published version may require journal subscription.

Published with permission from: Elsevier 


\section{PSYCHOMETRIC EVALUATION OF MULTIDIMENSIONAL PAIN \\ INVENTORY (SWEDISH VERSION) IN A SAMPLE OF ELDERLY PEOPLE}

Ulf Jakobsson, PhD, Senior lecturer

Vibeke Horstmann, PhD, Statistician

Department of Health Sciences, Faculty of Medicine, Lund University

P.O. Box 157, SE-221 00 Lund, Sweden

Address for correspondence;

Ulf Jakobsson, Department of Health Sciences, Faculty of Medicine, Lund University.

P.O. BOX 157, SE-221 00 Lund, Sweden.

E-mail: Ulf.Jakobsson@med.lu.se

Telephone: +46 4622219 24, Fax: +46 462221934

Number of text pages of the entire manuscript: 21

Number of tables: 7 


\section{ABSTRACT}

Objectives: The aim was to psychometrically evaluate the Swedish version of the Multidimensional Pain Inventory (MPI-S) and the "brief screening version of MPI-S” for use in an elderly sample.

Methods: This study comprised 175 people aged 76-99 years reporting pain and in need of help to manage daily living. The instrument's factor structures were investigated through factor analyses, convergent and discriminant validity were assessed through inter-scale correlations and correlations with items from SF-12. Reliability was assessed by Cronbach’s alpha.

$\underline{\text { Results: }}$ The full-length version of MPI-S did not, in general, show any satisfactory validity and reliability when used among elderly. It had acceptable convergent and discriminant validity, but the factor analysis did not show a good model fit. Low alpha values were found for most of the sub-scales. However, the brief screening version of MPI showed acceptable validity and reliability, except for rather low alpha values in section 3 and 4.

Conclusion: The MPI-S instrument may not be very useful for measuring pain among frail elderly. The brief screening version may instead be a better alternative to the full version of the MPI-S. However, the small number of observations may be the reason to the lack of fit, and further studies are warranted.

Key words: Aged, elderly, pain, pain measurement, psychometric evaluation, MPI-S 


\section{INTRODUCTION}

Assessment of pain is an essential part of nursing care. However, this assessment must be done with a psychometrically sound instrument for each specific group of patients (e.g. disease-specific, age-specific). Several instruments aimed at assessing pain have been developed over the years (Turk \& Melzack, 1992). One example of such an instrument is the West Haven-Yale Multidimensional Pain Inventory (WHYMPI) (Kerns et al., 1985) which measures pain from a multidimensional view. This instrument has been translated into Swedish and the Swedish version (MPI-S) has been psychometrically tested by Bergström et al. $(1998,1999)$. MPI-S has also been used to study pain in different patient groups characterised by: chronic musculoskeletal pain (Bergström et al., 1998, 1999), post-polio syndrome (Widar \& Ahlström, 1999) and stroke (Widar \& Ahlström, 2002). These studies included mostly middle-aged people. To the best of our knowledge there is no study that has psychometrically tested the instrument in an elderly sample, even though the instrument is likely to be useful for evaluating pain among these people. Thus, a study that psychometrically evaluates the MPI-S in an elderly sample is needed.

Based on WHYMPI, a brief version has also been developed (Kerns et al., 1985; Von Korff, 1992). This short version was constructed by choosing items from four subscales of the full-length instrument, aiming at giving a multidimensional view of the pain by using only a minimum of questions. The shorter version may be a good alternative to the full version of the WHYMPI, especially among elderly people who often do not have enough capacity, e.g. because of poor health, to answer comprehensive questionnaires. However, the brief version does not seem to have been

psychometrically evaluated at all in previous studies and therefore such analyses must 
be performed before the instrument can be taken into practice. In this present study a brief screening version has been constructed by taking the corresponding items from the MPI-S.

\section{AIM}

The aim was to psychometrically evaluate both the MPI-S instrument and the "brief screening version of MPI-S” for use among elderly people.

\section{METHODS}

Sample

This study comprised 175 people (aged 76-99 years) reporting pain and who were in need of help to manage activities of daily living (ADL). The sample was part of a larger study in southern Sweden with an age-stratified sample of people aged 75 years and older (cf. Jakobsson et al., 2003). The individuals in the larger study were randomly selected from the population (i.e. from a census list) for each age group: 75-79 ( $n=2500), 80-84(n=2500), 85-89(n=2000)$ and $90+$ years $(n=1500)$. Of a total of 8500 questionnaires, 4278 were returned in a usable form (mean age 83.7 years, SD 5.7 and $61.6 \%$ were women).

The respondents in the larger study were categorised in two groups, those in need of help with activities of daily living because of lowered health status (e.g. help with personal hygiene, getting dressed, cooking or preparing meals) and those who were not in need of help. The respondents in need of help $(n=1305)$ were contacted by phone and asked if they wanted to participate in a structured interview and 532 (41\%) agreed. 
Those who did not want to further participate in the interviews were significantly older $(p<0.001)$ than those who did participate further; however, there were no significant differences in the degree of pain between participants and non-participants. The interview took place where the respondent chose (mostly in their own homes) and further questions about e.g. their need of help to manage daily living, pain, pain management were asked. The questions in the interviews also covered demographic data and included a standardised instrument, MPI-S (Table 1). Those who reported having had pain for the last 3 months and answered the whole MPI-S questionnaire were included in the present study $(n=175)$. The study was approved by the Ethics Committee of the Medical Faculty at Lund University (LU 478-99).

\section{Measurement}

Multidimensional Pain Inventory - Swedish version (MPI-S) (Bergström et al., 1998) has been developed from the West Haven-Yale Multidimensional Pain Inventory (WHYMPI) (Kerns et al., 1985). WHYMPI contains 61 items distributed in 12 subscales, divided into three sections (Kerns et al., 1985). The Swedish version (MPI-S) includes 34 of the 61 items and only the first two sections of the WHYMPI (Bergström et al., 1998; 1999). MPI-S was psychometrically evaluated, among other tests, by a confirmatory factor analysis (LISREL) and showed the same factor structure as section 1 and 2 in other versions of the WHYMPI (Bergström et al., 1998). Each item has a seven-graded response-scale with fixed grading between 0 and 6, where 0 corresponds to "no, not at all” and 6 to "yes, very much”. The number of items in each sub-scales varies between two and nine. Section 1 contains (21 items) five sub-scales: pain severity, interference, life control, affective distress and support. In this present study, item number 13 in section 1 in MPI-S was removed because it was a question about 
work. Section two (12 items) focuses on the patient's perception of responses from significant others and consists of three sub-scales: punishing responses, solicitous responses and distracting responses. It should be noticed that only respondents with next-of-kin, e.g. spouses, can answer the items in section two. High scores in each subscale correspond to a high degree of e.g. pain severity, support, and punishing responses.

"Brief screening version of Multidimensional Pain inventory” (Kerns et al., 1985; Von Korff, 1992) is a shorter version of WHYMPI. The brief screening version consists of eight of the original items all selected from section 1 . The questions are two items from each of the first four factors, i.e. pain severity (items 1 \& 7), interference (items 4 \& 8), life control (items 15 \& 18), affective distress (items 20 \& 22).

Two items from SF-12 (Ware et al., 1996) were also used in this study. These two items were "During the past 4 weeks how much did pain interfere with your normal work (including work both outside the home and housework)?” (in this study called “SF12:1") and "How much time during the last month have you felt downhearted and low?" (in this study called "SF-12:2”). The responses for SF-12:1 ranged from "not at all” to "very much". The responses for SF-12:2 ranged from "not at all” to "all the time".

\section{Data analysis}

Spearman’s rank order correlation was used to assess convergent and discriminant validity of MPI-S as well as of the brief screening version. Convergent and discriminant 
validity were assessed by investigating inter-correlation between the sub-scales as well as correlation between the sub-scales and two items selected from SF-12.

To evaluate the MPI-S factor structures confirmatory factor analyses (LISREL 8.51) were performed, based on Spearman's rank order correlation matrix. Each of the two MPI-S sections was analysed separately. The factors were defined in concordance with the sub-scales in the instrument, while correlation between the latent variables, factor loadings on the latent variables, and the residuals were set as free parameters. As measures of fit for the factor model the chi-square goodness-of-fit test, the goodness-offit index (GFI), the adjusted goodness-of-fit index (AGFI), the normed fit index (NFI), and the non-normed fit index (NNFI) were used. If the factor model fits, the chi-square test should be non-significant (i.e. $\mathrm{p}>0.05$ ). The GFI, AGFI and NFI are scaled to range between 0 and 1 , with values above 0.90 suggesting a good model fit. An exception to this is the NNFI, which is not scaled to range between 0 and 1, although a higher value still indicates better fit. Unfortunately, when the number of free parameters increases in the model, the better it will fit e.g. in GFI (Nunnally \& Bernstein, 1994). However, the AGFI and the NNFI adjust (downwards) the GFI and NFI respectively, based on the number of parameters/degrees of freedom (Nunnally \& Bernstein, 1994).

To investigate the factor structure for the brief screening version of MPI-S an explorative factor analysis (varimax rotation) was performed. Some item responses (i.e. items 15 and 18) were revised so that all items ranged from positive to negative replies before the factor analysis was performed. Since the items in the instrument are chosen from four different sub-scales the number of factors to extract was set at four. Missing values were excluded pairwise. 
As reliability analysis, Cronbach’s alpha (Cronbach, 1951) was used to measure internal consistency. The alpha values preferably range between 0.7 and 0.9 even if figures as low as 0.6 may be acceptable (Nunnally \& Bernstein, 1994). Alpha values lower than 0.7-0.6 indicated too high heterogeneity and values higher than 0.9 indicate that the items may be too similar.

Data were analysed using SPSS for Windows 11.5 and LISREL 8.51.

\section{RESULTS}

The sample consisted of 175 people, aged 76-99, reporting chronic pain (Table 1). All respondents were, to some degree, in need of help with ADL. The mean duration of pain was 11.5 years (SD 15.6). Thirty-seven percent reported that they had been diagnosed, or at least knew the reason for the pain. The reasons reported were unspecified musculoskeletal pain (1\%), osteoporosis (2\%), rheumatoid arthritis (6\%), osteoarthritis (34\%), other rheumatic diseases (14\%) such as Sjögren’s syndrome, Systemic Lupus Erythematosus (SLE), fibromyalgia and unspecified rheumatic disease. Other reasons were musculoskeletal diseases/problems (27\%) such as fracture, displaced intervertebral disc, joint and muscle inflammation and other non-specified musculoskeletal problems. Non-musculoskeletal diseases/problems (16\%) as reasons for the pain included lowered circulation of the blood (above all in the legs), herpes zoster and damaged nerves. The locations of the pain were legs/feet (33\%), back (22\%), hip/pelvis (15\%), arms/hands (14\%), joints (6\%), the whole body (4\%) and other not specified (6\%). 
Validity and reliability of the MPI-S

Correlation between the five sub-scales in the MPI-S section 1 ranged between -0.021 and 0.501 (Table 2), and between 0.055 and 0.721 for the three sub-scales in section 2 (Table 3). Mostly all sub-scales were found to discriminate rather well, since the correlations were less than 0.40 and only two scales in section 1 (Table 2) and one in section 2 (Table 3) exceeded this value. Correlation between the two items from SF-12 and MPI-S indicated acceptable construct validity (Table 4). SF-12:1 correlated highly above all with Interference ( $\mathrm{r}=0.464)$ but also rather highly with Pain severity $(\mathrm{r}=0.310)$ and Life-control ( $\mathrm{r}=-0.238)$. SF-12:2 was highly correlated with Affective distress $(\mathrm{r}=0.410)$.

The factor model with factor loadings for section 1 of the MPI-S is presented in table 5 together with the description of each item. The chi-square test $(\mathrm{p}<0.001)$ provided by the LISREL program indicated that the fit of the factor model was not satisfactory (Table 5). GFI was 0.82 , AGFI was 0.77 , the NFI was 0.75 , and the NNFI was 0.81 , also indicating low goodness-of-fit. The confirmatory factor analysis for section 2 of the MPI-S could not be computed because Phi (i.e. the matrix of correlations between the latent variables) and Theta-Delta (i.e. the matrix of correlations between the variables and the residuals) were not positive definite.

Internal consistency for all scales showed alpha-values of 0.63-0.89 (Table $2 \& 3$ ). Only three (of five possible) sub-scales in section 1 and one (of three possible) in section 2 had alpha values over 0.7 (Tables $2 \& 3$ ). 
Validity and reliability of the "brief screening version of MPI-S"

The explorative factor analysis revealed a four-factor solution that explained about $61 \%$ of the total variance (Table 7). The factor structure followed the same factor structure as the MPI-S. The means (standard deviations) for the four factors were: $\mathrm{F} 1 \mathrm{~m}=2.62$ (1.56); F2 m=3.19 (2.17); F3 m=4.17 (1.50); F4 m=1.44 (1.64) (Table 1). Correlation between the two items from SF-12 and the brief screening version indicated, as for MPI-S, acceptable construct validity (Table 6). Cronbach’s alpha varied between 0.59 and 0.82 (Table 7). 


\section{DISCUSSION}

The MPI-S instrument did only show acceptable validity and reliability for some parts of the instrument and showed no satisfactory factor structure was found for neither section 1 or section 2 of the instrument. The brief screening version of MPI-S showed a factor structure similar to the MPI-S and acceptable validity and reliability. However, rather low reliability was found for the brief screening version in section 3 and 4 . Thus, the shorter version may be considered a better alternative to the full version of the MPIS when measuring pain among elderly.

The psychometric testing of the MPI-S instrument was done because the instrument has not been psychometrically evaluated before in studies focusing on elderly people. Furthermore, in this present study all elderly were in some kind in need of help with ADL, giving a sample of "frail elderly". Previous psychometric testing of MPI-S has only been done in a sample of middle-aged people with chronic musculoskeletal pain (Bergström et al., 1998; 1999). In this present study the instrument did not show acceptable validity and reliability, however, with some exceptions. Inter-scale correlations for section 1 and 2 (Table $2 \& 3$ ) showed mostly the same results as in previous studies (Kerns et al., 1985; Bergström et al., 1998; Widar \& Ahlström, 1999; 2002). Affective distress showed lack of significant correlation ( $\mathrm{r}=0.14)$ with pain severity, while in the study of Bergström et al. (1998) it showed a correlation of $r=0.36$ and in the studies of Widar \& Ahlström $(1999,2002)$ these two sub-scales correlated even more $(r=0.52-0.60)$. Furthermore, in this study and in both studies of Widar \& Ahlström (1999; 2002) a significant correlation were found between interference and support, while in the study of Bergström et al. (1998) a low ( $\mathrm{r}=0.10)$ correlation between these two scales was found, as in Kerns et al. (1985) (r=0.09). When the 
instrument was correlated with items from SF-12, it showed good convergent and discriminant validity (Table 4). Finally, the MPI-S instrument showed acceptable reliability only in some parts of the instrument (Tables 2 \& 3).

When the factor structure of the instrument was analysed, a non-acceptable model-fit was found for section 1 and section 2. Even if it is easier to get a significant chi-square test when using ordinal variables and when entering many variables in the factor analysis, as in this study, the other goodness-of-fit tests (GFI, AGFI, NFI, NNFI) did not show an acceptable model-fit. For section 2 no LISREL model could be computed at all. To further explore the factor structures explorative factor analyses were conducted for both sections of the instrument. For section 1 the same factor structure as in previous studies was found, but for section 2 the factor structure found was not at all like that in previous studies. Thus, only section 1 seems to be valid in terms of factor structure. Furthermore, it seems fair to assume that section 2 may not be very useful among elderly, especially among very old people. This because many of the respondents do not have any spouse or next-of-kin, and this section should then be deleted initially if the instrument is to be used in this group of people. The fact that 68\% of respondents did not have any spouse or next-of-kin, and hence did not respond to section 2, strengthens this assumption.

The brief screening version of MPI-S is probably a better alternative to the full version of MPI-S when measuring pain among elderly. The instrument can be seen as suitable for (frail) elderly people because it is short, easy to administer, easy to complete and still gives a overview of the elderly person's pain from a multidimensional perspective. The factor analysis showed the same factor structure as in the original instrument (MPI- 
S) and explained a rather large (61\%) part of the total variance (Table 7). Furthermore, the mean value and standard deviation for each factor were mostly the same as for the MPI-S (Table 1), and the short version showed acceptable convergent and discriminant validity. However, the Cronbach's alpha values were a little bit too low for factor 3 (alpha=0.59) and 4 (alpha=0.62), but may be considered as mostly acceptable. Thus, the brief screening version of MPI-S seems to be a valid and reliable instrument for measuring pain among elderly (especially when only using factors 1 and 2).

\section{Limitations}

The results must be seen in relation to the kind of sample the study used. In this study all the elderly were in need of help with activities of daily living due to poor health, hence are considered as frail elderly. Frail elderly are not well studied regarding pain and pain measurement. One explanation for this is that these frail elderly are a heterogeneous group, hence giving a great variation in their answers regarding their pain and its influence on daily life. This might in turn partly explain the low reliability and perhaps also the validity/factor structure found in this study. Low response rate was, as in other gerontological studies, also seen in this study with a $53 \%$ response rate in the first step of the larger population study and $41 \%$ in the "follow-up interviews". This magnitude of drop-outs is not uncommon in studies of elderly people, but still gives limitations in the external validity i.e. that the results may give a more positive view of the elderly's situation when the oldest and frailest are not included. Finally, in the confirmatory factor analysis there were few observations in relation to the number of parameters estimated. This might be one reason for the poor fit of the model. 


\section{CONCLUSION}

On the one hand, the MPI-S instrument did not seem to have sufficient validity and reliability for use in (frail) elderly. On the other hand, the brief screening version showed acceptable validity and is probably a better alternative to the full-length version although it had slightly too low reliability in half of the four sub-scales. Thus, the brief screening version of MPI-S seems to be a better alternative to the full version of MPI-S when measuring pain among elderly. However, more psychometric evaluation of the instruments is needed to further establish validity and reliability in different age- and patient groups.

\section{ACKNOWLEDGEMENTS}

The authors wish to thank all the respondents for participating in the study. We are also most grateful to Magdalena Andersson, Gunilla Borglin, Anna Ekwall, Ylva Hellström, Ann-Christine Janlöv, Karin Stenzelius, Bibbi Thomé for assistance with the data collection, and to Alan Crozier for his English revision of the manuscript. This study was supported by grants from the Swedish Rheumatism Association, the Vårdal Foundation and Department of Health Sciences, Faculty of Medicine, Lund University. 


\section{REFERENCES}

Bergström G, Jensen IB, Bodin L, Linton SJ, Nygren ÅL, Carlsson SG. Reliability and factor structure of the Multidimensional Pain Inventory - Swedish Language version (MPI-S). Pain 1998; 75: 101-110.

Bergström G, Jensen IB, Linton SJ, Nygren ÅL. A psychometric evaluation of the Swedish version of the multidimensional pain inventory (MPI-S): a gender differentiated evaluation. Europ J Pain 1999; 3: 261-273.

Cronbach LJ. Coefficient alpha and the internal structures of tests. Psychometrika 1951; 3: 297-334.

Jakobsson U, Klevsgård R, Westergren A, Hallberg IR. Old people in pain: a comparative study. J Pain Symptom Manage 2003; 26: 625-636.

Kerns RD, Turk DC, Rudy TE. The West Haven-Yale Multidimensional Pain Inventory (WHYMPI). Pain 1985; 23: 345-356.

Nunnally, JC. Bernstein, IH. (1994). Psychometric theory (3rd ed.). McGraw-Hill Inc. USA.

Turk DC, Melzack R. (ed.). Handbook of pain assessment. New York: The Guildford Press, 1992. 
Von Korff M. Epidemiological and survey methods: chronic pain assessment. In Turk DC, Melzack R. (ed.) Handbook of pain assessment. New York: The Guildford Press, 1992.

Ware J Jr, Kosinski M, Keller KD. A 12-item Short-Form Health Survey: construction of scales and preliminary tests of reliability and validity. Med Care 1996; 34: 220-233.

Widar M, Ahlström G. Pain in persons with post-polio. Scand J Caring Sci 1999; 13: $33-40$.

Widar M, Ahlström G. Disability after a stroke and the influence of long-term pain on everyday life. Scand J Caring Sci 2002; 16: 302-310. 
Table 1. Description of demographic data and MPI-S scores

Age, mean (SD)

Men/women \%

Living conditions \%

- Own homes

- $\quad$ Sheltered housing
$84.9(5.4)$

24.6 / 75.4

83.9

16.1

\section{MPI-S}

Section $1(\mathrm{n}=175)$

- Pain severity, mean (SD) 2.62 (1.56)

- Interference, mean (SD) $2.74(1.52)$

- $\quad$ Life control, mean (SD) 3.95 (1.40)

- Affective distress, mean (SD) 1.59 (1.43)

- Support, mean (SD) 3.79 (2.07)

Section $2(\mathrm{n}=52)$

- $\quad$ Punishing responses, mean (SD) $0.57(0.92)$

- $\quad$ Solicitous responses, mean (SD) 3.42 (1.32)

- $\quad$ Distracting responses, mean (SD) 3.18 (1.77)

\section{Brief screening version ${ }^{a}$}

- Pain severity, mean (SD)

- $\quad$ Interference, mean (SD)

$2.62(1.56)$

- $\quad$ Life control, mean (SD)

- $\quad$ Affective distress, mean (SD)

$4.17(1.50)$ $1.44(1.64)$

${ }^{\mathrm{a}}$ scores range between 0 and 6 (high scores indicate high degree of pain severity, interference, life control, affective distress, support) 
Table 2. Inter-correlations between the five factors in section $1(n=175)$

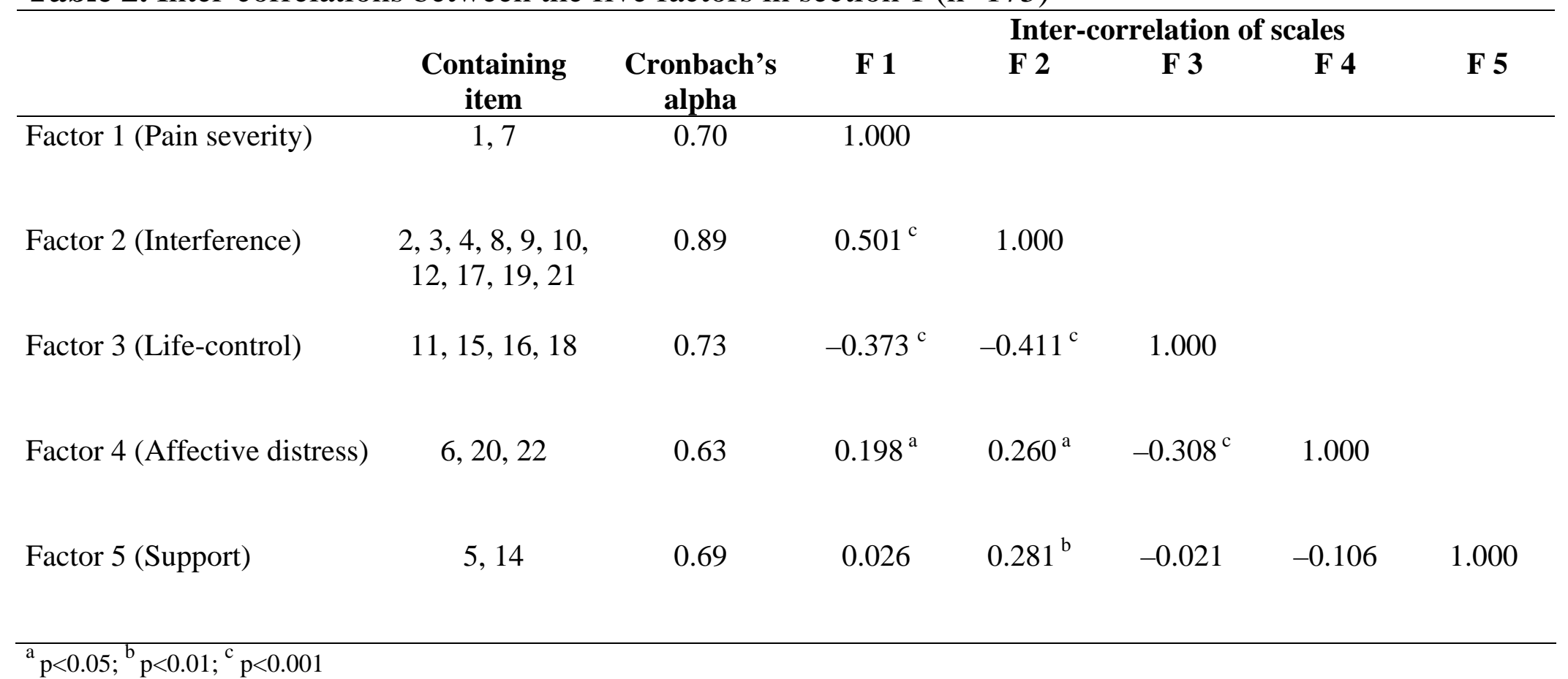


Table 3. Inter-correlations between the three factors in section $2(\mathrm{n}=52)$

Inter-correlation of scales

Containing Cronbach's $\quad$ F $6 \quad$ F 7

$$
\text { item }
$$

alpha

Factor 6 (Punishing responses)

2, 5, 8

0.70

1.000

Factor 7 (Solicitous responses)

$1,3,6,9,11$,

0.64

0.055

1.000

12

Factor 8 (Distracting

$4,7,10$

0.65

0.058

$0.721^{\mathrm{c}}$

1.000

responses)

${ }^{c} p<0.001$ 
Table 4. Correlations between items from SF-12 and MPI-S section $1(n=175)$ and section $2(n=52)$

\begin{tabular}{|c|c|c|c|c|c|c|c|c|}
\hline & $\begin{array}{c}\text { Pain } \\
\text { severity } \\
\text { (F1) }\end{array}$ & $\begin{array}{l}\text { Interference } \\
\text { (F2) }\end{array}$ & $\begin{array}{l}\text { Life-control } \\
\text { (F3) }\end{array}$ & $\begin{array}{l}\text { Affective } \\
\text { distress } \\
\text { (F4) }\end{array}$ & $\begin{array}{l}\text { Support } \\
\text { (F5) }\end{array}$ & $\begin{array}{l}\text { Punishing } \\
\text { responses } \\
\text { (F6) }\end{array}$ & $\begin{array}{l}\text { Solicitou } \\
\text { s } \\
\text { response } \\
\text { s } \\
\text { (F7) }\end{array}$ & $\begin{array}{l}\text { Distracting } \\
\text { responses } \\
\text { (F8) }\end{array}$ \\
\hline SF-12: 1 & $0.310^{* * *}$ & $0.464 * * *$ & $-0.238 * *$ & 0.133 & 0.045 & 0.039 & 0.191 & 0.013 \\
\hline SF-12 : 2 & -0.032 & -0.120 & 0.100 & $0.410 * * *$ & 0.010 & -0.134 & -0.139 & -0.199 \\
\hline
\end{tabular}

Table 6. Correlations between items from SF-12 and brief screening version

\begin{tabular}{|c|c|c|c|c|}
\hline & $\begin{array}{c}\text { Pain } \\
\text { severity }\end{array}$ & Interference & Life-control & $\begin{array}{c}\text { Affective } \\
\text { distress }\end{array}$ \\
\hline SF-12: 1 & $0.310 * * *$ & $0.382 * * *$ & $-0.175^{*}$ & 0.100 \\
\hline SF-12: 2 & -0.032 & -0.035 & 0.074 & $0.305^{* * *}$ \\
\hline
\end{tabular}


Table 5. Results of the confirmatory factor analysis (standardised solution) regarding the MPI-S section 1

\section{Scales and item}

Factor loadings

Pain severity

Rate the level of your pain at the present moment (Item 1)

On average, how severe has your pain been during the last week? (Item 7)

0.61

\section{Interference}

In general, how much does your pain interfere with your day-to-day activities? (Item 2)

Since the time your pain began, how much has your pain changed your ability to work? (Item 3 )

How much has your pain changed the amount of satisfaction or enjoyment you get from taking part in social and recreational activities? (Item 4)

How much has your pain changed your ability to participate in recreational and other social activities? (Item 8)

How much have you limited your activities in order to keep your pain from getting worse? (Item 9)

How much has your pain changed the amount of satisfaction or enjoyment you get from family-related activities? (Item 10)

How much has your pain changed your relationship with your spouse, family or significant other? (Item 12)

How much has your pain changed your ability to do household chores? (Item 17)

How much has your pain interfered with your ability to plan activities? (Item 19)

How much has your pain changed or interfered your friendship with people other than your family? (Item 21)

0.82

0.63

0.69

0.37

0.60

0.63

0.48

\section{Life control}

During the past week how much control do you feel that you have had over your life? (Item 11)

During the past week how much do you feel that you have been able to deal with your problems? (Item 15)

How much control do you feel that you have over your pain? (Item 16)

During the past week, how successful were you in coping with stressful situations in your life? (Item 18)

Affective distress

Rate your overall mood during the past week (Item 6)

During the past week how irritable have you been? (Item 20)

During the past week how tense or anxious have you been? (Item 22)

Support

How supportive or helpful is your spouse (or significant other) to you in relation to your pain? (Item 5)

How attentive is your spouse (or significant other) to you because of your pain? (Item 14) 
Table 7. Factor analysis (varimax rotation) for "the brief screening version of MPI-S".

\begin{tabular}{l|cccc|c}
\hline \multicolumn{1}{c|}{ Variable } & F 1 & F 2 & F 3 & F 4 & Communalities \\
\hline Item no. 1 & $\mathbf{0 . 8 3 9}$ & 0.201 & 0.000 & 0.161 & 0.770 \\
Item no. 7 & $\mathbf{0 . 5 2 9}$ & 0.421 & 0.135 & 0.100 & 0.458 \\
Item no. 4 & 0.212 & $\mathbf{0 . 8 0 7}$ & 0.066 & 0.049 & 0.702 \\
Item no. 8 & 0.160 & $\mathbf{0 . 8 0 5}$ & 0.158 & 0.099 & 0.709 \\
Item no. 15 & 0.310 & 0.105 & $\mathbf{0 . 4 0 0}$ & 0.298 & 0.356 \\
Item no. 18 & -0.015 & 0.164 & $\mathbf{0 . 8 8 4}$ & 0.159 & 0.835 \\
Item no. 20 & 0.067 & 0.138 & 0.052 & $\mathbf{0 . 7 9 5}$ & 0.659 \\
Item no. 22 & 0.152 & -0.008 & 0.233 & $\mathbf{0 . 5 2 4}$ & 0.352 \\
\hline Eigenvalues after rotation & 1.574 & 1.177 & 1.069 & 1.047 & \\
& & & & & \\
\% explained variance & 19.67 & 14.72 & 13.36 & 13.08 & \\
Cumulative \% & 19.67 & 34.39 & 47.75 & 60.84 & \\
& & & & & \\
Cronbach's alpha & 0.70 & 0.82 & 0.59 & 0.62 & \\
\hline
\end{tabular}

